\title{
Human Amniotic Fluid Stem Cells Modulate Muscle Regeneration After Cardiotoxin Injury in Mice
}

\author{
Silvia Zia ${ }^{1}$, Mattia Quattrocelli ${ }^{2}$, Ester Sara Di Filippo ${ }^{3,4}$, Nikhil Sindhwani $^{1}$, Francesca Bosisio ${ }^{5,6}$, Maurilio Sampaolesi ${ }^{2}$, Jan Deprest ${ }^{1,7 *}$ and \\ Jaan Toelen ${ }^{1,7}$
}

${ }^{1}$ Cluster Organ Systems, Department of Development and Regeneration KU Leuven, Leuven, Belgium

${ }^{2}$ Translational Cardiomyology Laboratory, Stem Cell Biology and Embryology Unit, Department of Development and Regeneration, KU Leuven, Leuven, Belgium ${ }^{3}$ Department of Neuroscience Imaging and Clinical Sciences, Interuniversity Institute of Myology (IIM), University "G.d'Annunzio" Chieti-Pescara, Chieti, Italy ${ }^{4}$ Center for Excellence on Ageing (CeSI), "G.d'Annunzio" Foundation, Chieti, Italy

${ }^{5}$ Laboratory of Translational Cell and Tissue Research, KU Leuven, Leuven, Belgium

${ }^{6}$ Department of Surgical Sciences, Milano-Bicocca State University, Monza, Italy

${ }^{7}$ Division Woman and Child, University Hospitals Leuven, Leuven, Belgium

\begin{abstract}
Amniotic fluid stem cells (AFSc) are a very heterogeneous subtype of stem cells with a broad multi potential. They could be used to treat congenital malformations or diseases. Recently, mesoangioblasts, resident pericytes of skeletal muscles, were shown to undergo muscle differentiation in vitro and in vivo. In this study we focused on the identification of an AFS subtype with pericytic characteristics and evaluate its myogenic potential.

We identified monoclonal AFSc lines expressing alkaline phosphatase activity (ALP) and the canonical pericytic markers neural-glial-2 chondroitin sulphate proteglycan (NG2), platelet derived growth factor receptor $\alpha$ and $\beta$ (PDGFR- $\alpha,-\beta$ ) and $\alpha$ smooth muscle actin ( $\alpha-S M A)$. These cells were able to integrate into the newly formed myotubes when co-cultured with the $\mathrm{C} 2 \mathrm{C} 12$ cells. To test the paracrine effects of these AFSC on muscle regeneration, we assessed their affects in a transwell assay with acutely injured myotubes. AFSc were able to modulate the expression of specific growth factors involved in muscle regeneration, such as Transforming Growth Factor $\beta$ (Tgf $\beta$ ), Interferon y (Ifny), Hepatocyte Growth Factor (Hgf) and Matrix Metalloproteinase 2 (Mmp2). When AFSc were injected in injured muscles they ameliorated muscle repair as measured by the reduction of centronucleated fibers and fibrosis. Interestingly, the transcriptional program of growth factor response in vitro is observed in large part in the in vivo xenograft experimental model, with the extension of Myostatin and Matrix Metalloproteinase 9 (Mmp9).
\end{abstract}

Our data suggest that AFSc subtype with pericytic characteristics have the ability to modulate muscle regeneration in vitro and in vivo.

Keywords: Amniotic fluid; Foetal stem cells; Perycite; Muscle regeneration; Stem cells

\section{Introduction}

In recent years, stem cells derived from amniotic fluid have drawn much attention in treatment of congenital and acquired perinatal conditions [1]. Amniotic fluid stem cells (AFSc) approximately constitute $1 \%$ of the cells found in the amniotic fluid (AF). AFSc are a heterogeneous population of cells with multipotent characteristics, retaining the ability to differentiate into different lineages such as adipogenic, chondrogenic, osteogenic and neurogenic [2-4]. The origin of these cells remains unknown. Several methods of AFSc isolation have been described and the isolation method probably influences the characteristics and differentiation potential of AFSs. AFSc can be cultured as a heterogeneous population [5] or isolated from early colonies consisting of two morphologically distinct adherent cell types, termed as spindle-shaped (SS) and round-shaped (RS) [6]. A small fraction of cells have been isolated for the expression of the marker c-Kit (stem cell factor receptor) [2] and shown to be positive for pluripotency markers [7-11]. AFSc integrated and participated in the regeneration of damaged organs, such as lung [12], kidney [13], heart $[14,15]$ and liver [16,17]. However, aforementioned methodologies do not distinguish among AFSc origins. A potential answer to this unsolved question is to derive a monoclonal population of stem cells by means of a single "starter cell" method. AFSc colonies derived from one single cell in primary culture were mechanically selected to derive a monoclonal population $[18,19]$. The advantage of this methodology is the possibility of obtaining a monoclonal population of cells at a very early stage in culture and of studying its origin. We recently described a monoclonal subtype of AFSc that expresses markers that are also found in the fetal urinary system [20]. These cells were able to attenuate the pathological changes of renal ischemia in a rat model.

The great potential of AFSc is the possibility of an autologous approach for congenital malformation or predictable neonatal acquired conditions, a research topic of our group. At present, Congenital Diaphragmatic hernia $(\mathrm{CDH})$ is one of the few conditions where the therapeutic potential of these cells has been investigated [21]. It is a malformation resulting in a diaphragmatic defect, with subsequent lung hypoplasia. Both these aspects could benefit from cellular therapy either by paracrine effects or by construction of a bioengineered patch for closure (necessary in around $40 \%$ of patients). Currently, synthetic and biodegradable patches are used to close the defect with high recurrence rates [22]. However, a tissue-engineered patch with

*Corresponding author: Jan Deprest, Division Woman and Child, University Hospitals Leuven, Leuven, Belgium, Tel: 0496239 992; E-mail: jan.deprest@uzleuven.be

Received April 27, 2016; Accepted May 17, 2016; Published May 25, 2016

Citation: Zia S, Quattrocelli M, Filippo ESD, Sindhwani N, Bosisio F, et al. (2016) Human Amniotic Fluid Stem Cells Modulate Muscle Regeneration After Cardiotoxin Injury in Mice. J Stem Cell Res Ther 6: 339. doi:10.4172/2157-7633.1000339

Copyright: () 2016 Zia S, et al. This is an open-access article distributed under the terms of the Creative Commons Attribution License, which permits unrestricted use, distribution, and reproduction in any medium, provided the original author and source are credited. 
addition of stem cells could provide a more effective and long-lasting solution $[23,24]$.

In this study, we focused on the identification of a AFSc pool suitable for muscle repair. Recently mesangioblast, a pericytic sub-population of mesenchymal-like cells that associated with the microvasculature wall, have been shown to be able to undergo the skeletal muscle lineage and restore muscle functionality $[25,26]$. Herein we demonstrate that some AFS pools show pericytic characteristics hence have the ability to integrate in myotubes in vitro and to improve muscle regeneration process in a mouse model of acute muscle damage in vivo.

\section{Materials and Methods}

\section{AFSc culture and characterization}

Human AFSc were derived and characterized following previously reported conditions [18]. Briefly, amniotic fluid (AF) was received from consenting women between 15-22 weeks of pregnancy (research program approved by the Ethics Committee of the UZ Leuven). AF was filtered with a $40 \mu \mathrm{m}$ strainer (BD bioscience, Erembodegem, Belgium) and centrifuged. The cell pellet was re-suspended in expansion medium consisting of a-MEM (Invitrogen, Ghent, Belgium), 15\% foetal bovine serum (Invitrogen, Ghent, Belgium), 1\% L-glutamine (Invitrogen, Ghent, Belgium), 1\% penicillin/streptomycin (Invitrogen, Ghent, Belgium) and 18\% Chang B and 2\% Chang C (Irvine Scientific, Brussels, Belgium), plated in a petri dish and incubated at $37^{\circ} \mathrm{C}$ in $5 \%$ $\mathrm{CO}_{2}$. As soon as single cells attached to the dish, medium was replaced to remove debris and unwanted epithelial cells. All individual cells were monitored daily using a light microscope. After a few days, single colonies were mechanically transferred into a 96 well plate and further expanded. AFSc from passage 4 in culture were characterized using flow cytometry analysis (FACS CantoI, BDbioscence, Erembodegem, Belgium) and tested for a complete mesenchymal stem cell CD marker panel consisting of CD117, CD24, CD90, CD44, CD105, CD73, HLA$\mathrm{ABC}$ (BD bioscience) and CD29 (Acris, Herford, Germany), HLA$\mathrm{DR}$ (BD bioscience) and for the hematopoietic markers CD34 and CD45 (BD bioscience) mouse anti-human monoclonal antibodies and appropriate isotype controls. The differentiation protocols were tested for different mesenchymal lineages (osteogenic, adipogenic and chondrogenic) [18].

Osteogenic differentiation: Osteogenic differentiation was induced by culturing AFSCs at $70 \%$ confluence for 4 weeks in 'osteogenic differentiation medium' (Invitrogen). Differentiation was assessed by Alizarin staining (Sigma Aldrich, Diegem, Belgium) of the calcified extracellular matrix deposition.

Adipogenic differentiation: To induce adipogenic differentiation, cells were cultured at $100 \%$ of confluence and subsequently differentiated with adipogenic differentiation medium for 14 days at $37^{\circ} \mathrm{C}$ in a $5 \% \mathrm{CO}_{2}$ incubator. This medium is composed of $10 \% \mathrm{FBS}$, $10^{-6} \mathrm{M}$ dexamethasone, $0.5 \mathrm{M}$ 3-isobutyl-methylxanthine, $10 \mathrm{mg} / \mathrm{mL}$ insulin, and $200 \mathrm{mM}$ indomethacin in Dulbecco's Modified Eagle Medium high glucose (Invitrogen). Differentiation into the adipogenic lineage was determined by Oil Red O staining (Sigma-Aldrich, MO, USA).

Chondrogenic differentiation: To induce chondrogenic differentiation, cells were cultured in high-density pellet mass cultures for 14 days. $20 \mathrm{~mL}$ droplets of cells suspension ( 400000 cells resuspended in phosphate buffered saline) were seeded into individual wells of a 24 well plate. Cells were allowed to attach without medium for $3 \mathrm{~h}$ at $37^{\circ} \mathrm{C}$ in a $5 \% \mathrm{CO}_{2}$ incubator and then cultured for $24 \mathrm{~h}$ in growth medium.
$24 \mathrm{~h}$ later, the medium was replaced with chondrogenic differentiation medium (Invitrogen). Chondrogenic differentiation was determined by Alcian Blue staining (Sigma-Aldrich).

Cell lines derived from different gestational weeks were tested for Alkaline Phosphatase activity by enzymatic colorimetric assay (SIGMAFAST BCIP/NBT tablet, Sigma Aldrich, Diegem, Belgium). Cells were grown at $100 \%$ of confluence and then fixed with $4 \%$ of PFA for 10 minutes. The staining was then performed following manufacturer's protocol. Signal was quantified using ImageJ software (Colour deconvolution plugin) in order to measure the percentage of the area stained.

To test whether AFSc share similar characteristics with pericytes, platelet derived growth factor receptor $\beta$ (PDGFR- $\beta$ ) (clone 958, Santa Cruz Biotechnology, Heidelberg, Germany) and a (Millipore, Overijse, Belgium), $\alpha$-smooth muscle actin (aSMA) (Dako, Heverlee, Belgium) and neural-glial-2 chondroitin sulphate proteglycan (NG2) (Millipore, Overijse, Belgium) were detected by immunofluorescence. For the immunofluorescence staining, cells were rinsed twice with cold PBS, and then subjected to fixation in 1\% PFA in PBS for $10 \mathrm{~min}$ at room temperature (RT). Cells were then permeabilized with a solution containing $0.2 \%$ triton and $2 \%$ BSA in PBS for $10 \mathrm{~min}$. Unspecific reactions were blocked with $10 \%$ donkey serum (Sigma Aldrich) in PBS for $20 \mathrm{~min}$. Cells were then incubated with primary antibody over night at $4^{\circ} \mathrm{C}$. After 3 washes $1 \%$ BSA in PBS, Alexa Fluor 594 and 488 secondary antibodies (Life technology, Merelbeke, Belgium) were incubated 1 hour at RT. Hoechst (Sigma Aldrich) was used for nuclei counterstaining.

\section{Manipulation of AFSC with lentiviral construct}

AFSC were manipulated with lentiviral vector $(\mathrm{LV})$ in order to express the reporter gene LacZ and GFP as previously described (18). Lentiviral production is explained elsewhere [27]. Briefly, lentiviral vector particles were added to the cell culture with a multiplicity of infection of 10. To detect transduction efficiency in LacZ-AFSC, X-gal staining was performed. Cells were fixed in $25 \%$ glutaraldehyde and $37 \%$ of methanol for 2 minutes and incubated with a X-gal solution (Sigma-Aldrich, Diegem, Belgium) overnight at $37^{\circ} \mathrm{C}$. Stained cells were counted using light microscopy and expressed as a 'percentage positive' in relation to the total cell population (\% positive cells). To detect GFP positive AFSC, flow cytometry analysis was performed. AFSC were trypsinezed, washed with PBS and immediately analysed with FACS Canto. Untransduced AFSC population was used a negative control to design proper gate.

\section{Differentiation towards muscle lineage}

Two populations of AFSc with pericyte characteristics were plated on a 24 well plate and grown till confluence. Then growth medium was replaced with differentiation medium (DMEM glutamax, sodium pyruvate $100 \mathrm{mM}$, insulin $10 \mu \mathrm{g} / \mathrm{mL}$, glutamine $1 \%$, transferrin $100 \mu \mathrm{g} /$ $\mathrm{mL}$, horse serum $5 \%$ and $1 \%$ penicillin and streptomycin; all reagents from Life Technology, Merelbeke, Belgium) and cells were kept for 7 days at $37^{\circ} \mathrm{C}$ in $5 \% \mathrm{O}_{2}$, changing medium every 3 days. To detect differentiation (myotubes formation), cultures were fixed with $1 \%$ PFA in PBS for 10 min at RT. Unspecific reactions were blocked with $10 \%$ donkey serum in PBS for $20 \mathrm{~min}$. Cultures were then incubated with primary antibody for Myosin heavy chain II (Mef20, DSHB) over-night at $4^{\circ} \mathrm{C}$. After 3 washes with $1 \%$ BSA in PBS, Alexa Fluor 594 secondary antibodies was incubated 1 hour at RT. Hoechst (Life technology, Merelbeke, Belgium) was used for nuclei counterstaining. 


\section{Co-culture with murine myogenic cell line $\mathrm{C} 2 \mathrm{C} 12$}

GFP- and LacZ-AFSc were co-cultured with murine skeletal myoblasts ( $\mathrm{C} 2 \mathrm{C} 12$ cells) in a ratio of $1: 3(\mathrm{C} 2 \mathrm{C} 12$ : $\mathrm{AFSc})$, and plated at 5,000 cells $/ \mathrm{cm} 2$ in AFSc growth medium.

Forty-eight hours after plating, cells reached $100 \%$ confluence and growth medium was shifted to differentiation medium (DMEM glutamax, sodium pyruvate $100 \mathrm{mM}$, insulin $10 \mu \mathrm{g} / \mathrm{ml}$, glutamine $1 \%$, transferrin $100 \mu \mathrm{g} / \mathrm{ml}$, horse serum $5 \%$ and $1 \%$ penicillin and streptomycin; all reagents from Life Technologies). Medium was replaced every 3 days until the end-point of 7 days. To detect differentiation, co-cultures with GFP-AFSc were fixed with $1 \%$ PFA in PBS for $10 \mathrm{~min}$ at RT. Unspecific reactions were blocked with $10 \%$ donkey serum in PBS for 20 min. Cultures were then incubated with primary antibody for myosin heavy chain II (Mef20, Developmental Studies Hybridoma Bank (DSHB), Iowa City, Iowa, USA) over-night at $4^{\circ} \mathrm{C}$. After 3 washes in 1\% BSA in PBS, Alexa Fluor 594 secondary antibody was incubated 1 hour at RT. Hoechst was used for nuclei counterstaining. Co-cultured with LacZ-AFSc and C2C12 were fixed in $25 \%$ glutaraldehyde and $37 \%$ methanol for 2 min and incubated with an X-gal solution (Sigma Aldrich) over-night at $37^{\circ} \mathrm{C}$ followed by eosin counterstaining. To detect myotubes structure, incubation with myosin heavy chain antibody was used as described above. Secondary antibody (goat anti rabbit PO, Dako) was incubated for 1 hour at RT at a concentration of 1:100. Biotin-conjugated secondary antibodies were detected with HRP-ABC signal amplification Kit followed by DAB detection. Micrographs of DAB-stained cells were directly taken with a phase contrast microscope (Leica).

\section{Myogenic modulation of AFSc in vitro}

To test the modulation capacity of AFSc on damaged $\mathrm{C} 2 \mathrm{C} 12$, a paracrine indirect co-culture assay was designed. C2C12 were seeded on a 24 well plate and cultured with differentiation medium (DMEM+2\%FBS+1\%PS) until myotubes were formed. After 7 days, cardiotoxin (CTX, $10 \mu \mathrm{M}$; Sigma-Aldrich) was added to the culture. After 24 hours, cells were washed with PBS and medium was replaced. In addition, Transwell ${ }^{\bullet}$ Permeable Supports (pore size $4 \mu \mathrm{m}$; Corning, Kruibeke, Belgium) with AFSc previously seeded was added to the culture. Cell seeding concentrations were selected in order for wells to reach confluence by day 1 of the experiment. Twenty-four and 48 hours later, C2C12 cells were harvested and RNA extracted to investigate expression of specific markers (Table 1).

LDH assay: To prove toxicity of CTX on C2C12 cells, an LDH assay (cat n. 88953, Thermo Scientific, Aalst, Belgium) was performed. C2C12 were plated and cultured for 7 days in DM until myotubes were formed. CTX $(10 \mu \mathrm{M})$ was added. Twenty-four hours later, the assay was performed following manufacturer's instruction.

Molecular analysis: RNA from $\mathrm{C} 2 \mathrm{C} 12$ cell line was extracted using TriPure reagent (Roche, Vilvoorde, Belgium) according to the manufacturer's instructions. cDNA was synthesized from $1 \mu \mathrm{g}$ of
RNA using Taq Man Reverse Transcription Reagents according to manufacturer's instructions (Applied Biosytem, Gent, Belgium). The SuperScript ${ }^{\text {tw }}$ III Platinum ${ }^{\circledR}$ SYBR $^{\circledR}$ Green One-Step qRT-PCR Kit with ROX (Invitrogen, Gent, Belgium) was used to detect gene expression in samples. Primer sequences are listed in Table 1. Gapdh was used as a reference gene to normalize mRNA levels and results were computed using the Delta Ct (Ct gene of interest - Ct GAPDH) formula and subsequently normalized to the level before differentiation.

\section{AFSc transplanted in CTX-injured skeletal muscle mice}

Animals were housed at the animalium of the Faculty of Medicine of the Catholic University of Leuven. Animals were kept at constant temperatures and humidity, with 12:12-h light-dark cycles and unrestricted access to standard diet and water. All experiments were performed in cohorts of aged-matched adult C57BL/6 mice. A total number of 24 animals was used for this experiment. Mice ( $\mathrm{n}=6 /$ group) were randomly assigned as follows: mice subjected to CTX injection into the right tibialis anterior (TA) and gastrocnemius (GSC) muscles and treated with PBS $(50 \mu \mathrm{L})$ (= group CTX+PBS); mice subjected to CTX injection into the right TA and GSC muscles and treated with AFSc (500.000 cells re-suspended in $50 \mu \mathrm{l}$ of PBS) (= group CTX + AFSc). Contralateral TA and GSC muscles were used as internal control (= group control). Mice were anesthetized with $1 \%$ Isofluorane (ISOVET, Eurovet, Heusden-Zolder, Belgium) and CTX (10 $\mu \mathrm{M}$; SigmaAldrich) was injected into the muscle belly of the TA and GSC of the right leg of the animal. Two days later, either PBS or AFSc were injected in the damaged area. Entire TA and GSC muscle, from tendon to tendon, were harvested at 1 and 2 weeks after injection. Excess fat and connective tissue were removed and muscles were quickly weighted. TA muscles were fixed in 4\% PFA and GSC muscles were snap-frozen for molecular analysis.

Histological evaluation: Muscle biopsies were fixed in $4 \%$ PFA, embedded into paraffin and then sectioned to $4 \mu \mathrm{m}$ slides and processed for H\&E staining. Fifteen non-overlapping fields were taken from muscle biopsies to count number of centrally nucleated fibers compared to the total number of fibers at 1 and 2 weeks.

Immunostaining for laminin was performed on paraffin slides. Briefly, sections were deparaffinized and endogenous peroxidase activity was blocked with $0.5 \% \mathrm{H}_{2} \mathrm{O}_{2}$ in $\mathrm{PBS}$ for $20 \mathrm{~min}$ at room temperature. Slides were then heated at $98^{\circ} \mathrm{C}$ for 1 hour in citrate buffer $(10 \mathrm{mmol} / \mathrm{L}, \mathrm{pH} 6.0)$ to enhance antigen retrieval. Non-specific binding was minimized by incubating sections in $2 \%$ BSA, $1 \%$ milk in PBS- $0.1 \%$ tween 80 for $30 \mathrm{~min}$. Sections were then incubated overnight at $4^{\circ} \mathrm{C}$ with the primary rabbit polyclonal antibodies against laminin at 1:100 dilutions (ab11575, ABcam, Cambridge, UK). Negative controls included buffer alone. Secondary antibody (goat anti rabbit HRP, Dako) was incubated at 1:100 dilutions for 1 hour. The color reaction was developed with 3,3'-diaminobenzidine (Sigma) and sections were counterstained with Mayer hematoxylin. Sections were then dehydrated through graded ethanol, cleared in xylene, and

\begin{tabular}{|c|c|c|}
\hline Gene & FW & RV \\
\hline Gapdh & CGACTTCAACAGCAACTCCCACTCTTCC & TGGGTGGTCCAGGGTTTCTTACTCCTT \\
\hline Hgf & CAACAGTAGGGTGGATGGTTAG & TGAGCCTTCAGGACCATAGA \\
\hline Ifn $\mathrm{\gamma}$ & TCAAGTGGCATAGATGTGGAAGAA & TGGCTCTGCAGGATTTTCATG \\
\hline Tgf $\beta 1$ & CGCAACAACGCCATCTATGA & CAAGGTAACGCCAGGAATTGT \\
\hline Myostatin & CAGGAGAAGATGGGCTGAATC & CCTGAGCAGTAATTGGCCTTA \\
\hline Mmp2 & CTACTTCTTCAAGGGTGCCTATT \\
\hline Mmp 9 & CCCTGCCAGTTTCCATTCA & CTGGAGAACAGTCAGCATCTA \\
\hline
\end{tabular}

Table 1: List of primers used to detect murine sequences. 
mounted in dePex (BDH, vWR International, Heverlee, Belgium). Ten non-overlapping fields were taken using a 200X magnification with a Zeiss microscope. Images were captured using the condenser phase contrast 1. Cross sectional fiber perimeter of each fiber were automatic measured with a MATLAB-based command line software. Software was specifically designed to measure cross sectional area (CSA) based on the work from Hodneland et al. [28]. The frequency distribution of single-fiber CSAs was determined. The single-fiber CSA was then divided into the total fiber CSA to provide an estimate of the number of fibers present in each muscle.

To detect fibrosis formation muscle section were stained for Masson's trichrome at 1 and 2 weeks after injury. The digital color images were segmented (color deconvolution plugin) and further binarized in order to measure the percentage of the fibrosis stained in blue.

Slides stained by H\&E and Masson Trichrome staining were evaluated by one researcher and an experienced pathologist (F.B) who were blinded to the sample identification.

PCR analysis: RNA from $\mathrm{C} 2 \mathrm{C} 12$ cell line or from the gastrocnemius muscles from the three different groups (control, $\mathrm{CTX}+\mathrm{PBS}, \mathrm{CTX}+\mathrm{AFSc}$ ) was extracted using TriPure (Roche) following the manufacturer's instructions. cDNA was synthetized and qPCR was performed for Myostatin, Ifn $\gamma, \operatorname{Hgf}$, Tgf $\beta 1$ and Mmp9. Primer sequences are listed in Table 1. Gapdh was used as a reference gene to normalize mRNA levels and results were computed using the Delta Ct (Ct gene of interest - Ct GAPDH) formula and subsequently normalized to the level before differentiation.

\section{Statistical analysis}

Results were expressed as mean \pm SD. Appropriate parametric or non-parametric statistical tests were chosen according to data distribution (Gaussian or not). qPCR analysis was analyzed using the t-test. Linear regression analysis was used to analyze the frequency of distribution of the fiber perimeter, while for the number of centronuclear fibers nonparametric Kruskal-Wallis followed by Dunn testing was used. Statistical significance level was defined as $\mathrm{p}<0.05$. Quantification of immunohistochemistry was analyzed using the t-test.

\section{Ethical approval}

AFSc isolation was approved by the UZ Leuven Medical Ethics committee (project number: ML4149). All procedures involving animals were approved by the Local Ethics Committee for Animal Experimentation of the Catholic University of Leuven (KU Leuven) (project number: P095/2012).

\section{Results}

\section{Some monoclonal AFSc lines express pericyte-like markers and have a myogenic differentiation potential in vitro}

Human monoclonal AFSc isolated from different harvest time points (15-21 weeks) invariably expressed canonical mesenchymal stem cell markers and were able to differentiate in 3 different mesenchymal lineages as previously shown (Figures S1 and S2) [18]. Some AFSc lines were variably positive to Alkaline Phosphatase activity (ALP; range: 0-56\%) (Figures 1A, S1(C) and Table 2). Within this population some AFSc lines expressed pericytic markers PDGFR $\beta$ and $\alpha$-SMA, and to a lower extent PDGFRa (Table 2). The pluripotency marker ALP was used as discriminating factor because by mesenchymal characteristics, differentiation potential or 'pericyte'-characteristics no ideal clone for muscle differentation was selected. To test the in vitro myogenic potential of these cells, two different cell lines with high expression of both pericytic markers and ALP activity were chosen (clone 1 and 2) (Figure S1). Moreover, these two specific cell lines had a high replication potential and could be expanded in culture for more than 30 passages. After 7 days under the myogenic differentiation protocol, few myotubes were observed in culture (Figure S3) showing a weak myogenic differentiation potential in vitro compared to the control murine cell line $\mathrm{C} 2 \mathrm{C} 12$ (Figure S3). Clone 1 and 2 were further cocultured with the murine cell line $\mathrm{C} 2 \mathrm{C} 12$ and integration of AFSc into the myotube structures was explored. In order to visualize the human cells, we used the AFSc that were labelled with the nLacZ or the eGFP. After 7 days of differentiation of the co-culture system LacZ-AFSc were detected in the newly formed myotubes. We were able to detect blue stained nuclei next to unstained nuclei in some myotubes when the $\mathrm{nLacZ}$ AFSc lines were used. An approximation of $5 \%$ of myotubes contained positive LacZ-AFSC were counted compared to the total number of myotubes formed. In the case of the eGFP-AFSc lines, we were able to visualize myotubes with a green cytoplasm (Figure 1).

\section{Myogenic modulation of AFSc in vitro}

In order to study a protective paracrine effect of AFSc on damaged myoblast, we set an in vitro study were $\mathrm{C} 2 \mathrm{C} 12$ were exposed to the toxic agent CTX and then cultured in a trans-well assay with AFSc. AFSc were able to modulate key gene expression in damaged $\mathrm{C} 2 \mathrm{C} 12$ cells. mRNA level of the inflammatory cytokine Ifn $\gamma$ was lower in $\mathrm{C} 2 \mathrm{C} 12$ in the presence of $\mathrm{AFSc}$, as compared to AFSc-free C2C12 culture, at 24 and 48 hours ( $\mathrm{p}=0.0022)$. Hgf and Tgf $\beta 1$ expression were also reduced at 48 hours $(\mathrm{p}=0.0022)$ while at $24 \mathrm{~h}$ the expression of this gene was still normal in both groups. During muscle regeneration, remodeling of the extracellular matrix is an important step. Matrix metalloproteinase Mmp2 is implicated in motility, differentiation and regeneration of skeletal muscle fibers. In our assay, mRNA of Mmp2 was drastically reduced at 48 hours in the trans-well culture compared to the control C2C12 ( 0.67 vs 37.37 fold increase, $\mathrm{p}=0.0022$ ) while no effect was seen at $24 \mathrm{~h}$ (Figure 2).

\begin{tabular}{|c|c|c|c|c|}
\hline CLONE & ALP (\%) & PDGFR $\beta$ & PDGFR $\alpha$ & $\alpha-S M A$ \\
\hline Clone 1 & 56.3 & + & + & ++ \\
\hline Clone 2 & 23.2 & ++ & - & +++ \\
\hline Clone 3 & 47.2 & + & - & + \\
\hline Clone 4 & 48.7 & + & + & ++ \\
\hline Clone 5 & 37.9 & + & - & +++ \\
\hline Clone 6 & 4.6 & ++ & - & - \\
\hline Clone 7 & 3 & + & - & +++ \\
\hline Clone 8 & 2.6 & + & + & ++ \\
\hline Clone 9 & 2.1 & + & - & - \\
\hline Clone 10 & 0.8 & + & - & - \\
\hline Clone 11 & 0 & ++ & - & - \\
\hline Clone 12 & 0.3 & nd & - & +++ \\
\hline Clone 13 & 0.4 & + & + & + \\
\hline Clone 14 & 0 & nd & - & + \\
\hline Clone 15 & 0 & nd & - & + \\
\hline
\end{tabular}

ALP is measured by $\%$ of positive stained area. For the expression of PDGFR- $\alpha / \beta$ and $\alpha$-SMA a score system was used to evaluate the presence of the markers visualized by IF: $(+) \sim 25 \%,(++) \sim 50 \%$ and $(+++)>50 \%$. Clone 1 and 2 were used for direct co-culture experiments. For the in vivo injection clone 1 was used.

Table 2: Analysis of pericyte markers on 15 different populations of AFSC 
Citation: Zia S, Quattrocelli M, Filippo ESD, Sindhwani N, Bosisio F, et al. (2016) Human Amniotic Fluid Stem Cells Modulate Muscle Regeneration After Cardiotoxin Injury in Mice. J Stem Cell Res Ther 6: 339. doi:10.4172/2157-7633.1000339
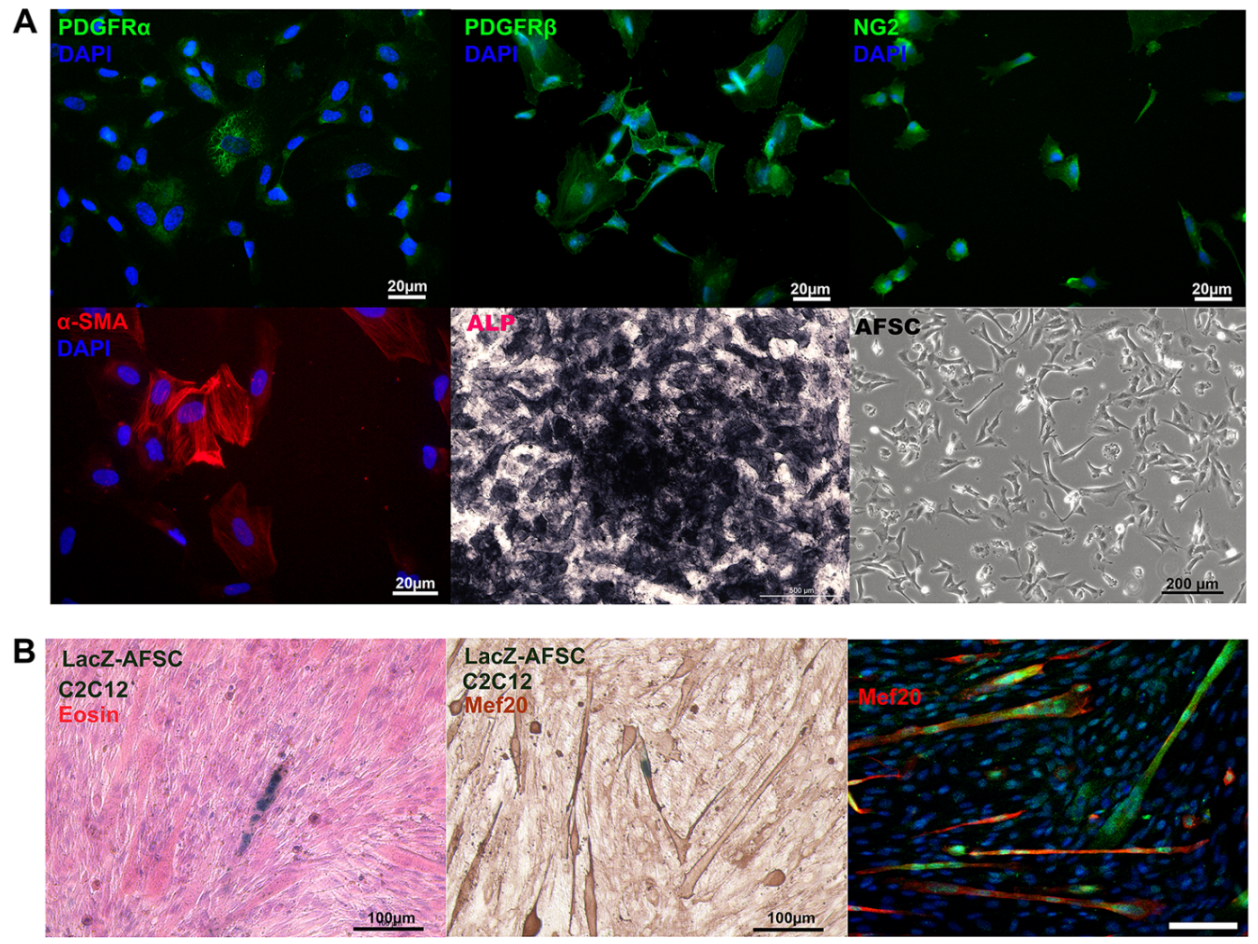

(A) Representative images of PDGFR $\alpha / \beta, \alpha-S M A, N G 2$ and for ALP activity in AFSC. (B) Nuclei from LACZ-AFSC are integrated into myotubes structure. Culture are counterstained with Eosin or with Mef20. In the right panel is shown the co-culture with GFP-AFSC with C2C12 after 7 days of differentiation.

Figure 1: Characterization of AFSC for pericytes markers and co-culture of AFSC with murine skeletal muscle cell line C2C12.

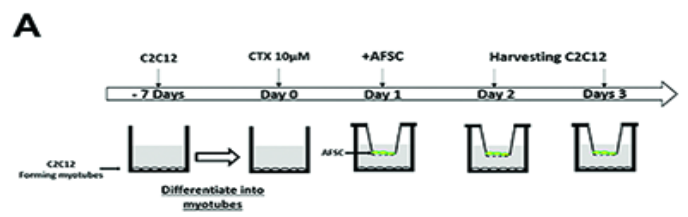

\section{B}

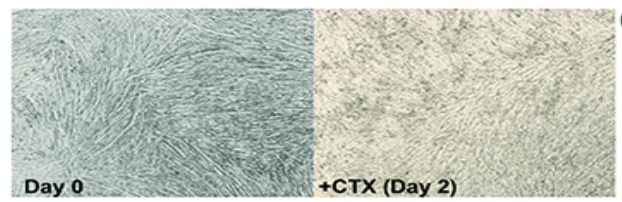

D
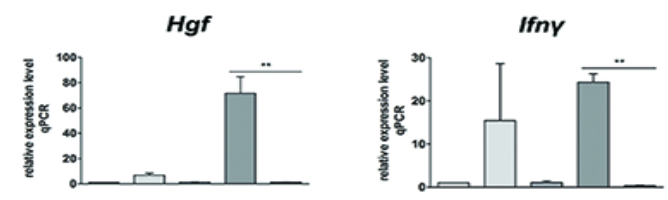

$\operatorname{Tgf\beta 1}$

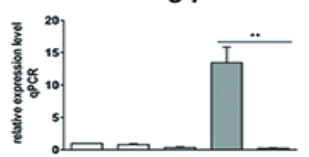

C

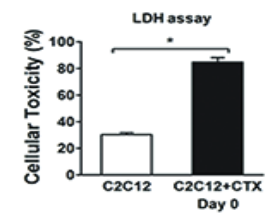

(A) Schematic representation of the experiment. (B) Representative figures of C2C12 myotubes at d0 and after 48 hours after incubation with Cardiotoxin. (C) LDH assay shows toxicity on $\mathrm{C} 2 \mathrm{C} 12$ cultures 24 hours after CTX exposure $(p=0.0238)$. (D) Molecular analysis of damaged myotubes after CTX exposition: $\mathrm{qPCR}$ analysis of $\mathrm{Hgf}$, Ifny, Tgf $\beta 1$ and Mmp2 expression in damaged myotubes with the addition of AFSC seeded in a trans-well. Three independent experiments were performed in triplicates and statistically analyzed using t-Test; Data are presented as mean $\pm S D ;{ }^{*} p=0,0238$. ${ }^{* *} p=0.022$.

Figure 2: In vitro assay to test paracrine effect of AFSC on damaged differentiated C2C12. 
A

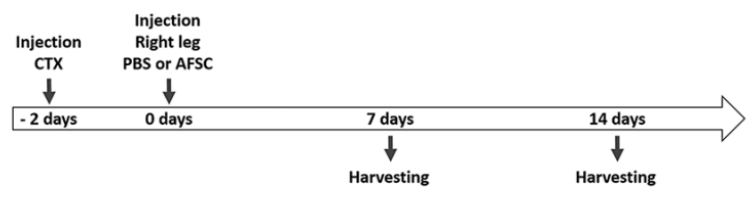

B

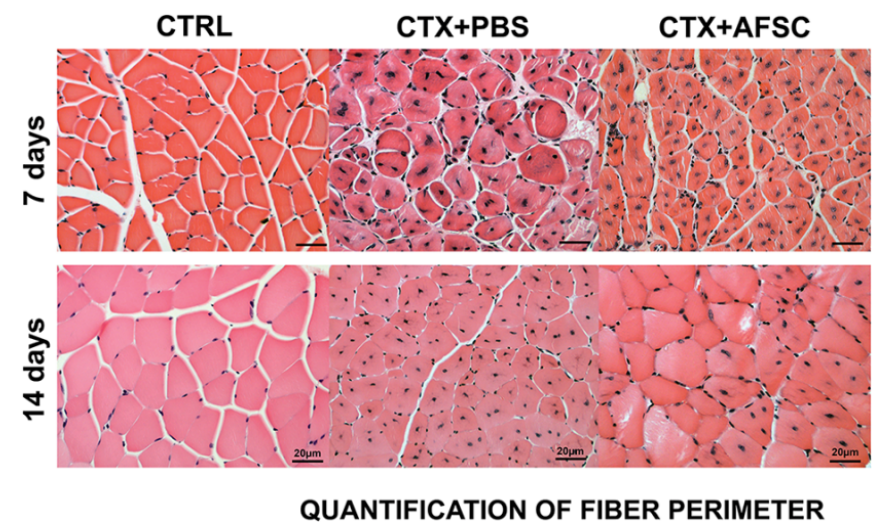
CENTRE NUCLETATED FIBERS

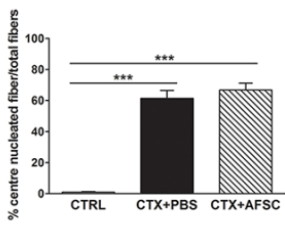

C
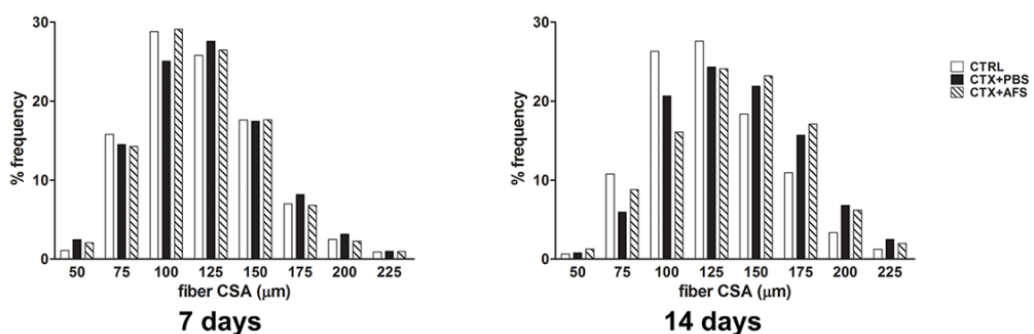

(A) Representative images of TA muscle sections for the 3 different groups (control, CTX+PBS and CTX+AFSC) stained for H\&E at 1 and 2 weeks. (B) Quantification of the CNF over total fibers $\left({ }^{* *} p<0.001 ;{ }^{* * *} p<0.0001\right)$. (C) Fiber percentage distribution among cross-sectional area (CSA) classes reveals a shift of AFSC-treated muscle toward higher CSA values.

Figure 3: Morphological analysis of tibialis anterior muscle sections.

\section{Pericyte-like AFSc improve muscle regeneration in CTX injured mice}

Having established that AFSc with pericytic characteristics have an in vitro paracrine effect in $\mathrm{C} 2 \mathrm{C} 12 \mathrm{CTX}$-injured culture, we subsequently investigated whether AFSc could have a regenerative potential in vivo in an acutely damaged muscle. 500,000 cells were injected into the muscle belly 48 hours after injection of CTX. Muscle biopsies were harvested 1 and 2 weeks after injection of cells.

Paraffin sections of TA muscles were stained with H\&E (Figure $3 \mathrm{~A}$ ) and number of centronuclear fibers were counted. The appearance of centrally nucleated fibers (CNFs) is a characteristic of regenerating myofibers. Ratio of CNFs were quantified as it is known that after acute muscle injury a better regeneration correspond to a faster disappearance of CNFs [29-31]. At 1 week no difference between the two groups was observed (CTX+PBS vs CTX + AFSc), while at 2 weeks $50 \%$ of the fibers were center nucleated in the AFSc-treated group. This was statistically different compared to the PBS-treated muscles ( 50.9 vs $80.87, \mathrm{p}<0.001$ ) (Figure 3B). Immunohistochemistry for laminin (Figure S4) was used for unbiased morphometric measurement. No statistical difference was observed in CSA of the muscle between groups (Figure 3C). At 2 weeks, a slight hypertrophy of the fibers in the AFSc treated animals was observed when compared to the PBS group. ASFC-treated animals had a higher frequency of fibers in the 150 and $175 \mu \mathrm{m}$ CSA categories as compared to PBS group (150: 23.2 vs $21.9 \mu \mathrm{m}$; 175: 17.1 vs $15.69 \mu \mathrm{m}$ ).

The injured muscles showed an inflammatory response in both groups (CTX+PBS and CTX+AFSc) with presence of inflammatory cells in the endomysium and surrounding the regenerating myocytes, as shown by their small size and the presence of centered nuclei (Figure S5). An inflammatory assessment by a blinded expert pathologist did not reveal any significant differences in inflammatory response between groups. Collagen deposition as a marker of early fibrosis was also observed in injured muscle. Slides stained for Masson's trichrome showed significant less fibrosis in the animals treated with AFSc compared to the PBS group (3.58 vs $16.65 \%$ area stained/HPF; p <0.001) (Figure 4). We investigated the possible paracrine mechanisms by which the AFSc could exert a therapeutic effect on the recovering muscle (Figure 4B). Levels of Tgf $\beta 1$, Hgf and Myostatin were down-regulated in animals treated with AFSc as compared to PBS group (Tgf $\beta 1: p=0.0036$; Hgf: $p=0.0022$; Myostatin: $\mathrm{p}=0.0022$ ). PBS-treated animals showed higher level of the cytokine Ifn $\gamma$ $(p=0.0337)$ and the matrix metalloproteinase $\operatorname{Mmp} 9(p=0.0281)$, when compared to the AFSc-treated group.

\section{Discussion}

In this study, we identified monoclonal populations of stem cells 
A

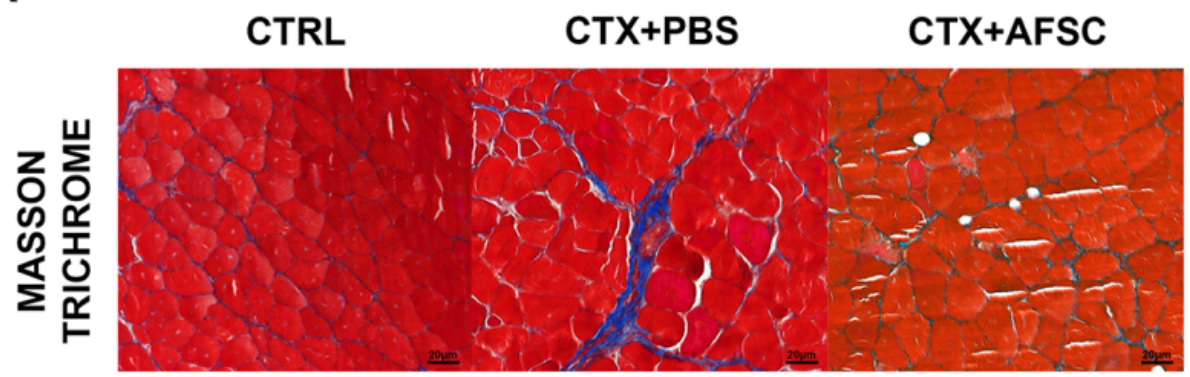

QUANTIFICATION

B

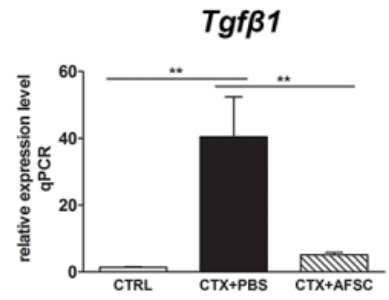

Hgf

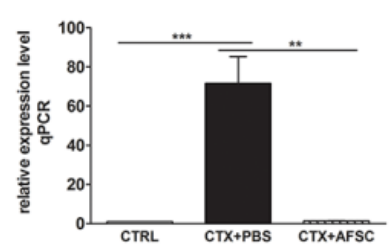

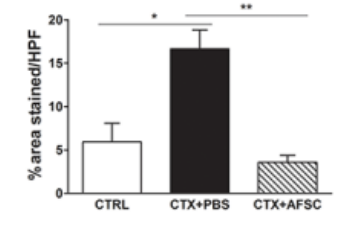

Mmp9
Myostatin

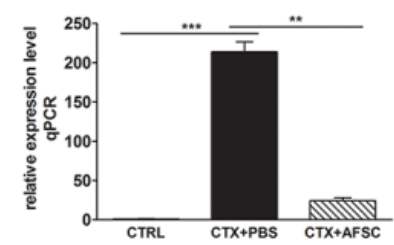

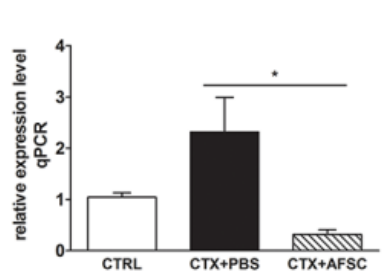

(A) Representative images of muscle sections stained for Masson's trichrome in all 3 groups at 1 week $\left({ }^{* *}<0.001 ;{ }^{* * *}, p<0.0001\right)$. (B) Molecular analysis of damaged muscle after CTX injection: qPCR analysis of Tgf $\beta 1$, Ifny, Myostatin, Hgf, Igf and Mmp2 mRNA expression in all 3 groups. Data were analyzed using t-Test; Data are presented as mean \pm SD; ${ }^{*} p<0.05$.

Figure 4: Histological analysis of injured skeletal muscle and possible paracrine effect of AFSC in muscle regeneration.

derived from amniotic fluid that ameliorates damaged myotubes in vitro and myofibers regeneration in vivo. Notably, we show that AFSc can modulate the expression of important factors for muscle growth and regeneration in vitro/in vivo in a paracrine manner. Our data contribute to the understanding of the potential of AFSc in injured skeletal muscles.

We previously showed that amniotic fluid contains cells that express markers that are suggestive for fetal renal origin. These cells were also able to attenuate the renal damage in an acute kidney injury model [20]. As we isolate the AFSC in such a manner that we are able to obtain monoclonal cell lines, we further investigated the entire AFSc population to see if other subtypes of cells were present. A certain subtype of these monoclonal AFSC express - next to the canonical mesenchymal stem cell markers [18] - markers that are also found in pericytes. The theory that pericytes are actually local muscle stem cells has gained more support in recent years [32,33]. These cells are defined as resident stem cells that can be activated and differentiated into a specific cell type after organ damage. In the skeletal muscle, when sorted for ALP, these cells are designated as mesoangioblasts [34] and can undergo skeletal myogenesis in vitro and in vivo [35,36]. We showed that a subset of AFSc express ALP and pericyte markers such as PDGFR- $\alpha$ and $\beta$ and $\alpha$-SMA. Even though these cells could only marginally be differentiated into myoblasts or myotubes, they were able to integrate into newly formed myotubes in vitro when co-cultured with murine myoblasts. Our results differ from previous reports [35], that showed myogenic differentiation of AFSc by culturing the cells with 5'-azacytidine. This resulted in an up-regulation of MYOD [35], yet the formation of myotubes in vitro was not shown. Our different findings may be attributed to differences in isolation and culturing conditions.

Since we were able to show the in vitro myogenic differentiation potential of AFSc, we also investigated their paracrine effect in vitro. We induced cell damage in a murine myoblast culture using CTX, then tested the indirect co-culture of AFSc with these murine $\mathrm{C} 2 \mathrm{C} 12$ cells. In our model, the addition of stem cells resulted in changes in selected factors that are thought to play a role in muscle proliferation and differentiation. High levels of Hgf in cultured myoblasts were earlier shown to reduce proliferation and differentiation [36,37]. The same effect was obtained with the over-expression of Tgf $\beta 1$ and Ifn $\gamma$ [38,39]. In our experiments, AFSc exert a contact-independent paracrine effect and modulate the expression levels of these factors. During regeneration, remodeling of the extracellular matrix that surrounds fibers is vital. Myoblasts can migrate into damaged areas and contribute to newly formed myofibers. The matrix metalloproteinase Mmp2 is constitutively expressed in muscle tissue, but after injury Mmp2 is transiently up-regulated and declines at 10 days post-injury 
and concomitantly with the generation of new fibers [40]. Intriguingly, addition of AFSc resulted in a restoration of Mmp2 expression. From this assay we can hypothesize that AFSc have a paracrine effect on injured $\mathrm{C} 2 \mathrm{C} 12$ and accelerate the healing process. The same pattern of expression was observed in CTX-damaged muscles of C57BL/6 mice. The mRNA expression levels of Myostatin, Ifn $\gamma$ and Tgf $\beta 1$ that decreased in the AFSc-treated group are considered a negative regulator of muscle growth, proliferation and regeneration [38]. In our study we saw a significant decrease of Hgf and Ifn $\gamma$ expression when animals were treated with AFSc. In accordance to our results, in literature it is known that Hgf factor is secreted from injured fibers and its level is decreasing over time [41], while Ifn $\gamma$ increased substantially following injury, peaking at 5 days post injury [42]. To strengthen the observation that AFSC accelerate muscle regeneration, animals treated with AFSc showed a decreased number of center nucleated fibers (CNFs) 14 days after treatment.

Fibrosis is the end of a cascade of events following tissue injury via inflammation, resulting in scar formation and potentially impairment of tissue function. Animals treated with AFSc showed a significant lower percentage of collagen deposition, which may indicate less final scarring. This observation could be correlated to the significant lower level of the pro-fibrotic factor Tgf $\beta 1$ and Myostatin in AFSc treated muscles. Myostatin has been proved to induce synthesis of ECM proteins, such as procollagen (types Ia1, I $\alpha 2$, and IIIa1) and to act synergically with Tgf $\beta 1$ to amplify the fibrotic process in injured or diseased skeletal muscles resulting in greater fibrosis than either could induce individually [43].

In this study we addressed a potential role of AFSc in muscle regeneration. Even though we observed a modulation of specific factors, the fine mechanisms of the putative short-term paracrine effects remain unknown. Despite AFSc are likely not directly committed toward the skeletal muscle lineage, we believe that they can exert a paracrine action that assists muscle regeneration or activates resident progenitor cells.

\section{Acknowledgment}

The Authors want to thank Rieta Van Bree and Catherina Luyten for the excellent technical assistance.

\section{References}

1. Gucciardo L, Lories R, Ochsenbein-Kolble N, Done E, Zwijsen A, et al. (2009) Fetal mesenchymal stem cells: isolation, properties and potential use in perinatology and regenerative medicine. BJOG 116: 166-172. [PubMed]

2. De Coppi P, Callegari A, Chiavegato A, Gasparotto L, Piccoli M, et al. (2007) Amniotic fluid and bone marrow derived mesenchymal stem cells can be converted to smooth muscle cells in the cryo-injured rat bladder and prevent compensatory hypertrophy of surviving smooth muscle cells. J Urol 177: 369376. [PubMed]

3. Wei PC, Chao A, Peng HH, Chao AS, Chang YL, et al. (2014) SOX9 as a Predictor for Neurogenesis Potentiality of Amniotic Fluid Stem Cells. Stem Cells Transl Med 3: 1138-1147. [PubMed]

4. Maraldi T, Bertoni L, Riccio M, Zavatti M, Carnevale G, et al. (2014) Human amniotic fluid stem cells: neural differentiation in vitro and in vivo. Cell Tissue Res 357: 1-13. [PubMed]

5. Bossolasco P, Montemurro T, Cova L, Zangrossi S, Calzarossa C, et al. (2006) Molecular and phenotypic characterization of human amniotic fluid cells and their differentiation potential. Cell Res 16: 329-336. [PubMed]

6. Roubelakis MG, Bitsika V, Zagoura D, Trohatou O, Pappa KI, et al. (2011) In vitro and in vivo properties of distinct populations of amniotic fluid mesenchymal progenitor cells. J Cell Mol Med 15: 1896-1913. [PubMed]

7. Moschidou D, Drews K, Eddaoudi A, Adjaye J, De Coppi P, et al. (2013) Molecular signature of human amniotic fluid stem cells during fetal development. Curr Stem Cell Res Ther 8: 73-81. [PubMed]
8. Moschidou D, Mukherjee S, Blundell MP, Drews K, Jones GN, et al. (2012) Valproic acid confers functional pluripotency to human amniotic fluid stem cells in a transgene-free approach. Mol Ther 20: 1953-1967. [PubMed]

9. Moschidou D, Mukherjee S, Blundell MP, Jones GN, Atala AJ, et al. (2013) Human mid-trimester amniotic fluid stem cells cultured under embryonic stem cell conditions with valproic acid acquire pluripotent characteristics. Stem Cells Dev 22: 444-458. [PubMed]

10. Zani A, Cananzi M, Fascetti-Leon F, Lauriti G, Smith VV, et al. (2014) Amniotic fluid stem cells improve survival and enhance repair of damaged intestine in necrotising enterocolitis via a COX-2 dependent mechanism. Gut 63: 300-309. [PubMed]

11. Wolfrum K, Wang Y, Prigione A, Sperling K, Lehrach H, et al. (2010) The LARGE principle of cellular reprogramming: lost, acquired and retained gene expression in foreskin and amniotic fluid-derived human iPS cells. PloS one 5 e13703. [PubMed]

12. Carraro G, Perin L, Sedrakyan S, Giuliani S, Tiozzo C, et al. (2008) Human amniotic fluid stem cells can integrate and differentiate into epithelial lung lineages. Stem Cells 26: 2902-2911. [PubMed]

13. Perin L, Sedrakyan S, Giuliani S, Da Sacco S, Carraro G, et al. (2010) Protective effect of human amniotic fluid stem cells in an immunodeficient mouse model of acute tubular necrosis. PLoS One 5: e9357. [PubMed]

14. Bollini S, Cheung KK, Riegler J, Dong X, Smart N, et al. (2011) Amniotic fluid stem cells are cardioprotective following acute myocardial infarction. Stem Cells Dev 20: 1985-1994. [PubMed]

15. Kim SW, Zhang HZ, Kim CE, Kim JM, Kim MH (2013) Amniotic mesenchymal stem cells with robust chemotactic properties are effective in the treatment of a myocardial infarction model. Int J Cardiol 168: 1062-1069. [PubMed]

16. Zheng YB, Zhang XH, Huang ZL, Lin CS, Lai J, et al. (2012) Amniotic-fluidderived mesenchymal stem cells overexpressing interleukin-1 receptor antagonist improve fulminant hepatic failure. PLoS One 7: e41392. [PubMed]

17. Liu H, Liu DQ, Li BW, Guan LD, Yan ZF, Li YL, et al. (2011) Human amniotic fluid-derived stem cells can differentiate into hepatocyte-like cells in vitro and in vivo. In Vitro Cell Dev Biol Anim 47: 601-618. [PubMed]

18. Zia S, Toelen J, Mori da Cunha M, Dekoninck P, de Coppi P, et al. (2013) Routine clonal expansion of mesenchymal stem cells derived from amniotic fluid for perinatal applications. Prenat Diagn 33: 921-928. [PubMed]

19. Phermthai T, Odglun $Y$, Julavijitphong $S$, Titapant $V$, Chuenwattana $P$, et al. (2010) A novel method to derive amniotic fluid stem cells for therapeutic purposes. BMC Cell Biol 11: 79. [PubMed]

20. da Cunha MGMCM, Zia S, Oliveira Arcolino F, Carlon MS, Beckmann DV et al (2015) Amniotic Fluid Derived Stem Cells with a Renal Progenitor Phenotype Inhibit Interstitial Fibrosis in Renal Ischemia and Reperfusion Injury in Rats. PLoS One 10: e0136145. [PubMed]

21. Pederiva F, Ghionzoli M, Pierro A, De Coppi P, Tovar JA (2013) Amniotic fluid stem cells rescue both in vitro and in vivo growth, innervation, and motility in nitrofen-exposed hypoplastic rat lungs through paracrine effects. Cell Transplant 22: 1683-1694. [PubMed]

22. Tsai J, Sulkowski J, Adzick NS, Hedrick HL, Flake AW (2012) Patch repair for congenital diaphragmatic hernia: is it really a problem? J Pediatr Surg 47: 637-641. [PubMed]

23. Kunisaki SM, Fuchs JR, Kaviani A, Oh JT, LaVan DA, et al. (2006) Diaphragmatic repair through fetal tissue engineering: a comparison between mesenchymal amniocyte- and myoblast-based constructs. J Pediatr Surg 41 : 34-39. [PubMed]

24. Deprest J, Gucciardo L, Eastwood P, Zia S, Jimenez J, et al. Medical and regenerative solutions for congenital diaphragmatic hernia: a perinatal perspective. Eur J Pediatr Surg 24: 270-277. [PubMed]

25. Dellavalle A, Maroli G, Covarello D, Azzoni E, Innocenzi A, et al. (2011) Pericytes resident in postnatal skeletal muscle differentiate into muscle fibres and generate satellite cells. Nat Commun 2: 499. [PubMed]

26. Dellavalle A, Sampaolesi M, Tonlorenzi R, Tagliafico E, Sacchetti B, et al. (2007) Pericytes of human skeletal muscle are myogenic precursors distinct from satellite cells. Nat Cell Biol 9: 255-267. [PubMed]

27. Ibrahimi A, Velde GV, Reumers V, Toelen J, Thiry I, et al. (2009) Highly efficient multicistronic lentiviral vectors with peptide $2 A$ sequences. Hum Gene Ther 20 845-860. [PubMed] 
Citation: Zia S, Quattrocelli M, Filippo ESD, Sindhwani N, Bosisio F, et al. (2016) Human Amniotic Fluid Stem Cells Modulate Muscle Regeneration After Cardiotoxin Injury in Mice. J Stem Cell Res Ther 6: 339. doi:10.4172/2157-7633.1000339

28. Hodneland E, Kogel T, Frei DM, Gerdes HH, Lundervold A (2013) CellSegm - a MATLAB toolbox for high-throughput 3D cell segmentation. Source Code Biol Med 8: 16. [PubMed]

29. Kim MH, Kay DI, Rudra RT, Chen BM, Hsu N, et al. (2011) Myogenic Akt signaling attenuates muscular degeneration, promotes myofiber regeneration and improves muscle function in dystrophin-deficient $\mathrm{mdx}$ mice. Hum Mol Genet 20: 1324-1338. [PubMed]

30. Guardiola O, Lafuste P, Brunelli S, laconis S, Touvier T, et al. (2012) Cripto regulates skeletal muscle regeneration and modulates satellite cell determination by antagonizing myostatin. Proc Natl Acad Sci U S A 109: E3231- E3240. [PubMed]

31. Toschi A, Severi A, Coletti D, Catizone A, Musaro A, et al. (2011) Skeletal muscle regeneration in mice is stimulated by local overexpression of $\mathrm{V} 1 \mathrm{a}$ vasopressin receptor. Mol Endocrinol 25: 1661-1673. [PubMed]

32. Bianco P, Robey PG, Simmons PJ (2008) Mesenchymal stem cells: revisiting history, concepts, and assays. Cell Stem Cell 2: 313-319. [PubMed]

33. Bianco P (2011) Back to the future: moving beyond "mesenchymal stem cells". J Cell Biochem 112: 1713-1721. [PubMed]

34. Quattrocelli M, Palazzolo G, Perini I, Crippa S, Cassano M, et al. (2012) Mouse and human mesoangioblasts: isolation and characterization from adult skeletal muscles. Methods Mol Biol 798: 65-76. [PubMed]

35. De Coppi P, Bartsch G Jr, Siddiqui MM, Xu T, Santos CC, et al. (2007) Isolation of amniotic stem cell lines with potential for therapy. Nat Biotechnol 25: $100-$ 106. [PubMed]

36. Gal-Levi R, Leshem Y, Aoki S, Nakamura T, Halevy O (1998) Hepatocyte growth factor plays a dual role in regulating skeletal muscle satellite cell proliferation and differentiation. Biochim Biophys Acta 1402: 39-51. [PubMed]

37. Yamada M, Tatsumi R, Yamanouchi K, Hosoyama T, Shiratsuchi S, et al. (2010) High concentrations of HGF inhibit skeletal muscle satellite cell proliferation in vitro by inducing expression of myostatin: a possible mechanism for reestablishing satellite cell quiescence in vivo. Am J Physiol Cell Physio 298: C465-C476. [PubMed]

38. Charge SB, Rudnicki MA (2004) Cellular and molecular regulation of muscle regeneration. Physiol Rev 84: 209-238. [PubMed]

39. Londhe P, Davie JK (2011) Gamma interferon modulates myogenesis through the major histocompatibility complex class II transactivator, CIITA. Mol Cell Biol 31: 2854-2866. [PubMed]

40. Kherif S, Lafuma C, Dehaupas M, Lachkar S, Fournier JG, et al. (1999) Expression of matrix metalloproteinases 2 and 9 in regenerating skeletal muscle: a study in experimentally injured and mdx muscles. Dev Biol 205: 158170. [PubMed]

41. Suzuki S, Yamanouchi K, Soeta C, Katakai Y, Harada R, et al. (2002) Skeletal muscle injury induces hepatocyte growth factor expression in spleen. Biochem Biophys Res Commun 292: 709-714. [PubMed]

42. Cheng M, Nguyen MH, Fantuzzi G, Koh TJ (2008) Endogenous interferongamma is required for efficient skeletal muscle regeneration. Am J Physiol Cell Physiol 294: C1183-C1191. [PubMed]

43. Zhu J, Li Y, Shen W, Qiao C, Ambrosio F, et al. (2007) Relationships between transforming growth factor-beta1, myostatin, and decorin: implications for skeletal muscle fibrosis. J Biol Chem 282: 25852-25863. [PubMed] 